Génét. Sél. Evol., 1985, 17 (1), 1-24

\title{
La différenciation des caryotypes polytènes dans le genre Bilobella (Collembola Neanurinae)
}

\author{
P. CASSAGNAU, L. DEHARVENG et N. PEJA * \\ Laboratoire de Zoologie, L.A. 333. Ecobiologie des Arthropodes édaphiques \\ Université P. Sabatier, 118 , route de Narbonne, F 31062 Toulouse Cedex \\ * Institut de Zoologie, Université de Tirana (Albanie)
}

\begin{abstract}
Résumé
Nous comparons les caryotypes polytènes de 7 espèces du genre Bilobella. L'analyse des séquences de bandes révèle des différences interspécifiques considérables qui contrastent avec la relative similitude des phénotypes externes. Il est en particulier impossible de reconnaître tous les chromosomes homologues d'espèce à espèce et nous avons du distinguer 11 types différents de chromosomes dont la combinaison conduit au caryotype haploïde $\mathrm{n}=7$ dans chaque espèce. L'étude morphométrique des caryotypes apporte quelques résultats mais jusqu'à présent seuls les caractères morphologiques externes ont permis de reconstituer une phylogenèse satisfaisante du genre. Une telle situation apparaît complètement différente de celle connue chez les diptères à chromosomes polytènes et pose le problème de la signification génétique de la disposition des bandes sur les chromosomes géants des collemboles Neanurinae.
\end{abstract}

Mots clés : Collemboles, cytogénétique, polyténie, phylogénie.

\section{Summary}

The differentiation of the polytene karyotypes in the genus Bilobella (Collembola Neanurinae)

We compare the polytene karyotypes of 7 species of the genus Bilobella. The analysis of the band sequences displays large interspecific differences, in contrast with the relative similarity of the external phenotypes. It is more particularly impossible to homologize all chromosomes through the seven species, and it is necessary to bring out eleven distinct types of chromosomes the combination of which leads to an haploid karyotype $\mathbf{n}=7$ in every species.

The morphometric study of the karyotypes gives some results, but so far, only the characters of external morphology have allowed satisfying phylogenetic reconstruction of the genus. Such a situation is completely different from the one which is known in Diptera with polytene chromosomes, and states the problem of the genetic significance of the chromosomal band sequences in the chromosomes of Neanurinae Collembola.

Key words : Collembola, cytogenetics, polyteny, phylogeny. 


\section{Introduction}

Le genre Bilobella Caroli, 1912, tel qu'il a été redéfini par l'un de nous (CAssAGNAU, 1979) présente ce que l'on pourrait appeler une "structure de distribution " assez classique chez les Collemboles Neanurinae, avec d'une part une espèce largement expansive (Bilobella aurantiaca) qui peuple toute la région méditerranéenne occidentale, d'autre part 11 espèces à distribution restreinte, localisées à l'est du Rhône avec un centre de diversité maximum au nord de l'Albanie (fig. 1).

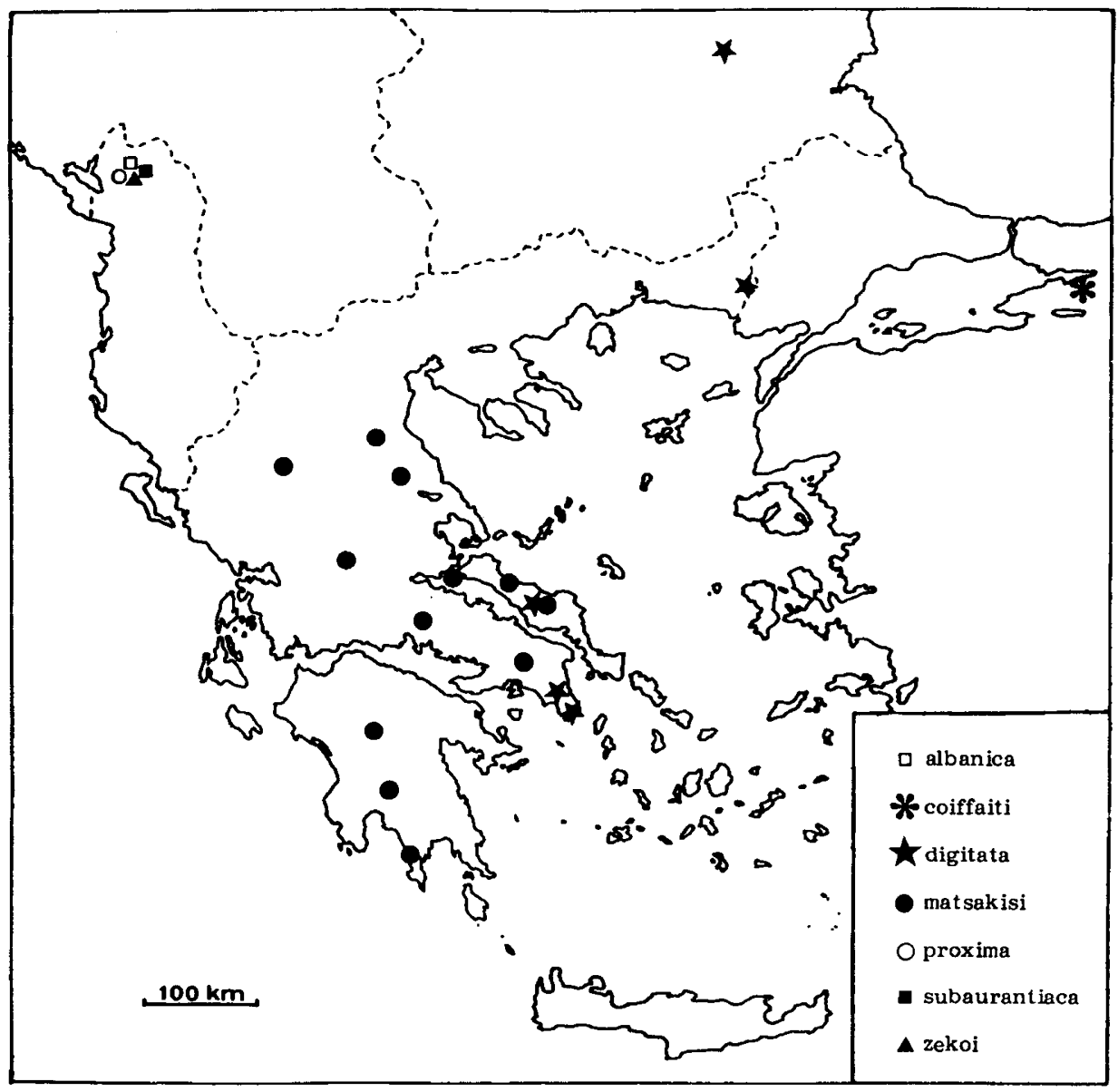

FIG. 1

Répartition des espèces de Bilobella en Europe méditerranéenne orientale.

Repartition of the Bilobella species in eastern Mediterranean Europe. 


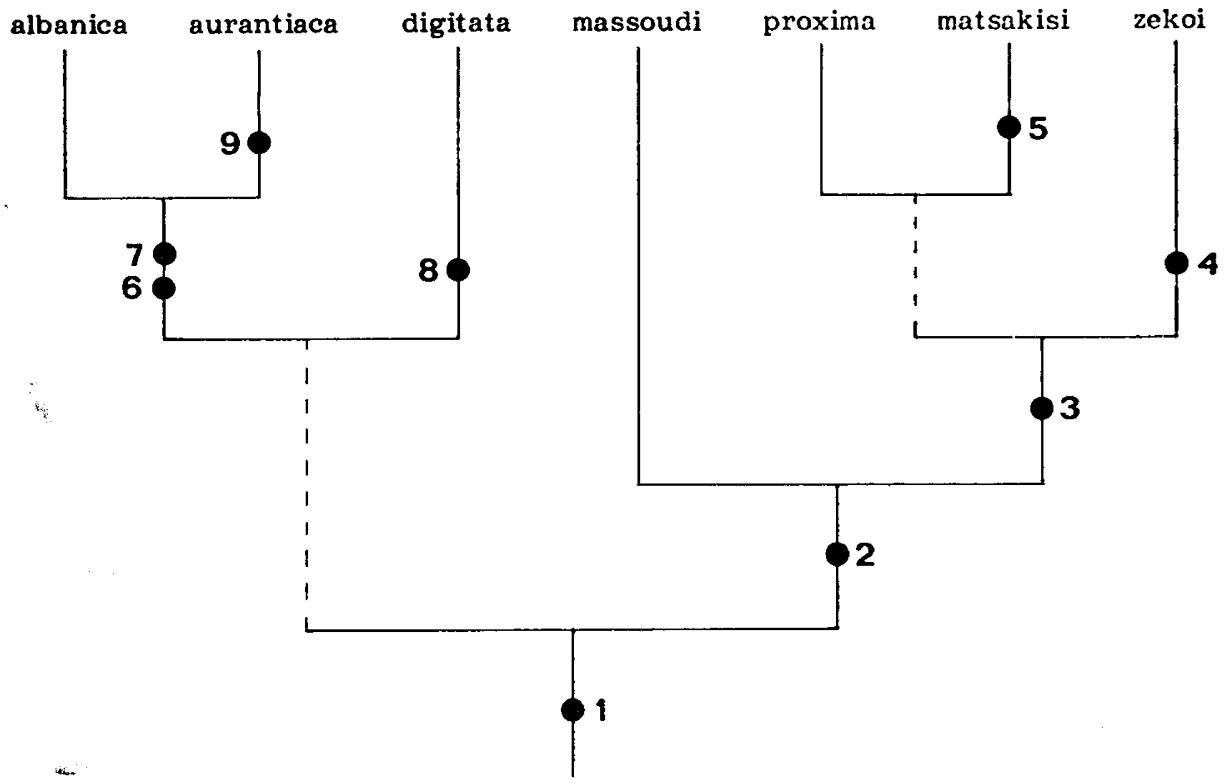

Fig. 2

Hypothèse phylogénétique des filiations dans le genre Bilobella établie sur la base des principales synapomorphies.

Phylogenetical hypothesis of the filiations in the genus Bilobella, based on the main synapomorphies.

1. Disparition de la soie et du tubercule dorso-internes sur th. I. Loss of the dorso-internal seta and tubercule on th. $I$.

2. Plurichaetose au niveau du tubercule L d'abd. V (5 à 26 soies). Plurichaetosis on the $L$ tubercule of abd. $V$ (5 to 26 setae). chétotaxie primitive).

3. Tubercules Dl de th. II et III avec 3 soies (perte d'une soie par rapport à la chétotaxie primitive.

Dl tubercles of th. II and III with 3 setae (I seta lost with regard to the primitive chaetotaxy).

4. Tubercules Dl d'abd. I à III avec 4 soies (1 soie supplémentaire par rapport à la chétotaxie primitive).

Dl tubercles of abd. I to III with 4 setae (1 supplementary seta with regard to the primitive chaetotaxy).

5. Tubercules L d'abd. I à III avec 6 à 9 soies (3 ou 4 soies dans la chétotaxie primitive).

$L$ tubercles of abd. I to III with 6 to 9 setae (only 3 or 4 in the primitive chaetotaxy).

6. Tubercules De de th. II et III avec 3 soies (perte d'une soie par rapport à la chétotaxie primitive).

De tubercles of th. II and III with 3 setae (1 seta lost with regard to the primitive chaetotaxy).

7. Tubercule $\mathrm{L}$ soudé au tubercule $(\mathrm{Dl}+\mathrm{De}+\mathrm{Di})$ sur abd. V.

$L$ tubercles united with $(D l+D e+D i)$ on abd. $V$.

8. Tubercules allongés et digités.

Tubercles elongated in finger shape.

9. Absence de la soie $O$ céphalique.

Cenhalic $O$ seta absent.

En pointillés : synapomorphies incertaines.

Datted line : uncertain synapomorphies. 
Du point de vue taxonomique, les caractères différentiels utilisés sont d'ordre morphologique. La chétotaxie dorsale présente des différences spécifiques particulièrement discriminantes qui sont à la base de la systématique du genre et permettent, moyennant certaines hypothèses, de proposer des affinités interspécifiques conformes à la figure 2 .

Les Bilobella constituant un matériel particulièrement favorable aux études cytogénétiques, nous nous sommes alors demandés si l'analyse chromosomique ne permettrait pas d'obtenir des indications plus précises sur la filiation des espèces, comme pouvaient le laisser présager les recherches classiques menées par ailleurs sur les Diptères à caryotype polytène (KEYL, 1959-1961; Dunbar, 1959 ; Lemeunier, 1979). Nous avions (CASSAgnau, $1968 \mathrm{a}, 1970 \mathrm{~b}, 1976$ ) déjà fait connaître le caryotype des espèces aurantiaca, massoudi et matsakisi. Nous avons également réuni des données inédites pour 4 autres espèces (albanica, digitata, proxima et zekoi) si bien que notre analyse chromosomique prendra en compte 7 espèces sur les 12 que compte le genre Bilobella.

\section{Matériel et méthodes}

\section{A. Animaux}

Les Bilobella, comme presque tous les Neanurinae, sont des Collemboles peu mobiles, dépourvus de furca et inféodés aux milieux forestiers, ou subforestiers. On les récolte à vue dans les humus ou sous les fragments de bois tombés à terre, à l'aide de pinceaux fins. Très hygrophiles, ils sont placés dans des tubes garnis de plâtre humidifié et ramenés vivants au laboratoire.

L'extraction en laboratoire à l'aide d'appareils de Berlese à partir de prélèvements d'humus ou de mousses peut aussi donner de bons résultats, mais fournit surtout des individus jeunes peu propices aux manipulations ultérieures.

\section{B. Méthodes cytogénétiques}

Les individus adultes (de 2 à $3 \mathrm{~mm}$ ) sont fixés au Carnoy pendant 24 heures. Cette fixation est indispensable compte tenu de la fragilité des glandes salivaires. On sectionne le corps en arrière de la première paire de pattes, les glandes pénétrant dans le prothorax. Les glandes salivaires sont ensuite disséquées sur lame à l'aide de minuties, dans une goutte d'acide acétique à $50 \mathrm{p}$. 100 légèrement glycériné. L'excès d'acide acétique est éliminé à l'aide d'un tampon de papier Joseph et remplacé par une goutte d'orcéine acéto-lactique. L'intensité de la coloration est contrôlée à la loupe binoculaire. Quand les chromosomes sont manifestement bien colorés, on recouvre d'une lamelle carrée dont le poids suffit en général à étaler convenablement les bras chromosomiques. Un léger choc contre le bord de la lamelle permet souvent de parfaire cet étalement.

L'orcéine est ensuite aspirée à l'aide d'un tampon de papier Joseph, appliqué étroitement contre un bord de la lamelle cependant qu'est disposée contre le bord opposé une goutte de gomme arabique phéniquée. La substitution de l'orcéine par la 
gomme se poursuit progressivement, en chambre humide, la lamelle étant maintenue en place par une masselotte de plomb posée sur sa surface. L'opération est achevée au bout de quelques heures. Il suffit alors de faire sécher à l'air libre la préparation qui peut se conserver ainsi plusieurs années. L'avantage de cette méthode est que l'on travaille toujours en milieux aqueux, ce qui évite les diverses manipulations de déshydratation des méthodes classiques, qui sont préjudiciables pour les chromosomes beaucoup plus fragiles que ceux des Diptères.

Seuls sont pris en considération les noyaux des lobes g.p2 et g.p3 (CASSAGNaU, 1968) où le degré de polyténie élevé permet l'analyse ultérieure des caryotypes.

\section{Résultats}

Le caryotype de chaque espèce comporte un nombre haploïde de chromosomes égal à 7. Nous les avons numérotés en chiffres romains de I à VII à l'intérieur de chaque espèce. La première espèce analysée a été Bilobella aurantiaca, où les chromosomes ont été ordonnés suivant leur longueur. La numérotation des chromosomes des autres espèces s'est faite chaque fois que cela a été possible par comparaison avec le caryotype de référence de $B$. aurantiaca. Mais dans bien des cas, comme nous le verrons plus loin, les homologies n'ont pas été possibles et nous avons dû envisager une numérotation globale en chiffres arabes des types chromosomiques rencontrés parmi les 49 chromosomes étudiés, soit au total 11 grands types irréductibles l'un à l'autre dans l'état actuel de nos connaissances. Ils sont schématisés sur la figure 3. Sur les planches 1 à 4 nous avons indiqué le numéro d'ordre dans le caryotype (I à VII, en bas) et le numéro du type (1 à 11 , en haut).

\section{A. Les types chromosomiques (fig. 3 et tabl. 1)}

\section{Type 1}

Le type chromosomique 1 se retrouve dans toutes les espèces étudiées. Il s'agit d'un métacentrique (parfois acrocentrique) de grande taille, atteignant 27,6 à 43,4 p. 100 de la longueur totale du caryotype haploïde. Le rapport de la longueur des deux bras euchromatiques varie de 1,15 à 2,86 . Le type 1 ne présente jamais de puffs bien développés, mais parfois un fort renflement à la base du bras long ( $B$. massoudi).

\section{Type 2}

Il est également bien reconnaissable. C'est un long chromosome nettement acrocentrique $(15,5$ à 25,1 p. 100 du caryotype haploïde et bras long/bras court $=1,59$ à 3$)$. Le bras long présente généralement une petite houpette ou un renflement terminal, sauf chez B. albanica. Chez B. digitata, aucun chromosome n'a pu être rapporté au 2.

\section{Type 3}

Il se trouve dans toutes les espèces sauf $B$. albanica, avec des variations relativement peu importantes de forme et de longueur $(11,0$ à 20,9 p. 100 du caryotype 
haploïde). Il s'agit d'un télocentrique ou subtélocentrique, le bras court n'étant, dans ce dernier cas, jamais nettement structuré.

\section{Type 4}

Ce chromosome très semblable au 3 chez aurantiaca et digitata est également présent chez albanica (télo- ou subtélocentrique, atteignant 9,2 à 15,4 p. 100 du caryotype haploïde). On note généralement la présence d'un petit puff subterminal caractéristique. Le type 4 n'a pas d'équivalent dans les autres espèces du genre.

\section{Types 5 et $5^{\prime}$}

Ce sont des chromosomes de taille moyenne, métacentriques ou plus rarement acrocentriques $(6,3$ à 13,6 p. 100 du caryotype haploïde, bras long/bras court $=1,4$ à 3,3 ). Un nombre variable de renflements ou de puffs sont développés sur chacun des bras. Le type chromosomique 5 existe chez toutes les espèces. Le type $5^{\prime}$ est peut-être dérivé du 5 par un remaniement chromosomique du type inversion péricentrique du segment $\mathrm{a}-\mathrm{b}$, associé à des variations plus fines au niveau des bandes (pl. 4). Les types 5 et $5^{\prime}$ sont tous deux curieusement présents dans la garniture haploïde de $B$. zekoi.

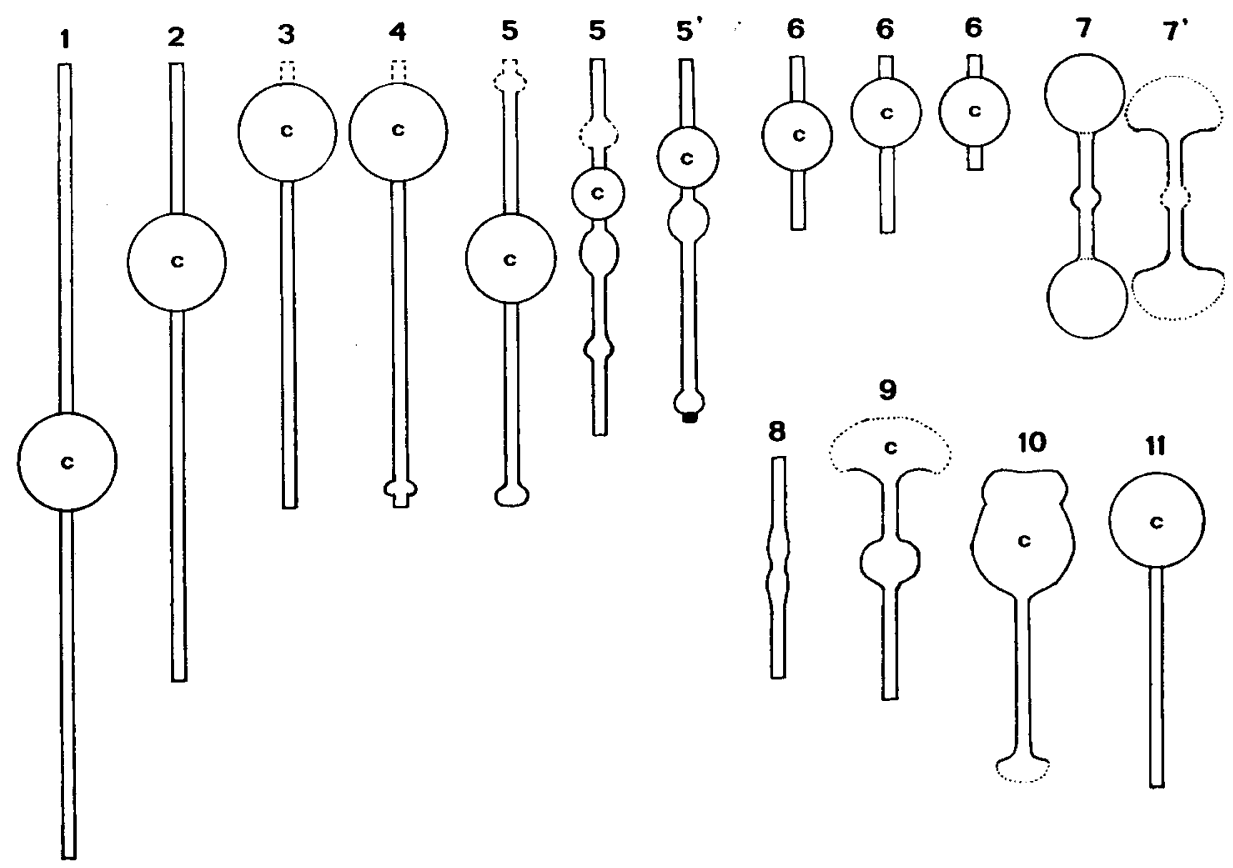

FIG. 3

Schématisation des types chromosomiques reconnus dans le genre Bilobella. Schematization of the chromosomal types recognized in the genus Bilobella.

c : Région centromérienne.

Centromeric region. 


\section{TABLEAU 1}

Les types chromosomiques chez les 7 espèces étudiées du genre Bilobella.

The chromosomal types in the 7 species of the genus Bilobella under study.

\begin{tabular}{|c|c|c|c|c|c|c|c|c|c|c|c|c|c|}
\hline & 1 & 2 & 3 & 4 & 5 & $5^{\prime}$ & 6 & 7 & $7^{\prime}$ & 8 & 9 & 10 & 11 \\
\hline aurantiaca & I & II & III & IV & V & $\ldots$ & VI & VII & - & - & - & - & - \\
\hline albanica & I & II & - & IV & III & 一 & ? VI & - & - & V & - & VII & - \\
\hline massoudi ...... & I & II & IV & 一 & ? III & - & VI & - & - & - & V & VII & - \\
\hline digitata . & I & - & III & IV & $\mathrm{V}$ & - & VI & - & - & - & 一. & VII & II \\
\hline proxima & I & II & IV & - & VI & - & III & - & V & - & - & VII & - \\
\hline matsakisi & I & II & IV & - & VI & 一 & III & - & V & - & - & VII & 一 \\
\hline zekoi & I & II & III & - & IV & IV & VI & - & - & - & - & VII & - \\
\hline
\end{tabular}

Les différents types sont décrits dans le texte et illustrés dans la figure 3 . La nomenclature en chiffres latins est expliquée dans le texte.

The different types are described in the text and illustrated on figure 3. Roman figure nomenclature is explain in the text.

\section{Type 6}

Malgré un fort polymorphisme intraspécifique, ce chromosome se retrouve facilement chez toutes les espèces examinées. Il reste court $(4,0$ à 8,9 p. 100 du caryotype haploïde), mais la longueur relative de ses bras varie beaucoup (bras long/bras court $=1,0$ à 3,7$)$.

\section{Types 7 et $7^{\prime}$}

Ils correspondent à des chromosomes courts $(5,0$ à 7,7 p. 100 du caryotype haploïde) caractérisés par la présence d'une large écharpe à chaque extrémité. Dans le type 7 propre à $B$. aurantiaca, les écharpes sont ovoïdes alors qu'elles forment des houppes à contour imprécis chez proxima et matsakisi. Aucun chromosome ne correspond à ce type dans les autres espèces.

\section{Type 8}

Ce type chromosomique très particulier n'est rencontré que chez $B$. albanica. Il se présente sous la forme d'un court segment euchromatique régulièrement structuré, atteignant 7,8 p. 100 de la longueur totale du caryotype haploïde.

\section{Type 9}

Le type 9 rappelle un peu les types 3,4 ou 11, mais il en diffère par la présence d'un gros renflement ovoïde médian. La zone centromérienne se termine en large houppe floconneuse. Ce chromosome atteint 12,0 p. 100 du caryotype haploïde et n'existe que chez $B$. massoudi. 


\section{Type 10}

La morphologie du type 10 est très caractéristique : c'est un télocentrique court ( 2,7 à 7,6 p. 100 du caryotype haploïde) dont le bras euchromatique se termine en houppette ou renflement (avec un nodule hétérochromatique chez $B$. zekoi). L'extrémité de la région centromérienne porte toujours un anneau ou un nodule d'hétérochromatine. Le type 10 existe chez toutes les espèces à l'exception de $B$. aurantiaca.

\section{Type 11}

La particularité essentielle du caryotype de B. digitata est de renfermer un télocentrique assez long en plus des types télocentriques 3 et 4 , pour lequel nous avons dû admettre un onzième type chromosomique. Il atteint 9,8 p. 100 du caryotype haploïde, et pourrait, éventuellement, correspondre à une scission du chromosome de type 2 absent chez cette espèce.

\section{Remarques générales}

1. Les « écharpes * sont des zones où la coalescence des chromatides est faible, la texture du chromosome polytène y devenant lâche. On y reconnaît cependant l'alternance régulière de bandes chromophiles et d'interbandes, avec parfois de larges plages hétérochromatiques très colorables. Elles sont souvent très développées au niveau des zones centromériennes. Elles n'ont pas la structure typique des anneaux de Balbiani, mais peuvent peut-être jouer le même rôle, en particulier lors des mues où elles deviennent très floconneuses et perdent leurs alternances de bandes. Il y a été localisé des $A R N$ après injection d'uridine tritiée, mais toute l'expérimentation reste à faire. Elles ne sont pas sans rappeler les zones élargies des chromosomes polytènes de certains Diptères (Simuliidae, Trypetidae...).

2. Il ressort des données exposées (fig. 3 et tabl. 1) que certains types chromosomiques se retrouvent, dans leurs grands traits structuraux, chez toutes les espèces (types 1,5 et 6 ), ou chez la majorité d'entre elles (types 2, 3 et 10). D'autres sont limités à 1 espèce (types $5^{\prime}, 7,8,9$ et 11), 2 espèces (type $7^{\prime}$ ) ou 3 espèces (type 4).

Les comparaisons interspécifiques révèlent un couple matsakisi-proxima dont tous les types chromosomiques ont pu être homologués. Un niveau d'affinités plus faible ( 6 types chromosomiques en commun : 1, 2, 3, 5, 6 et 10) existe entre les 2 espèces, massoudi et zekoi. Enfin les 3 espèces aurantiaca, albanica et digitata se montrent plus éloignées à la fois entre elles et avec chacune des autres espèces, ce que l'on peut illustrer par la représentation schématique suivante :

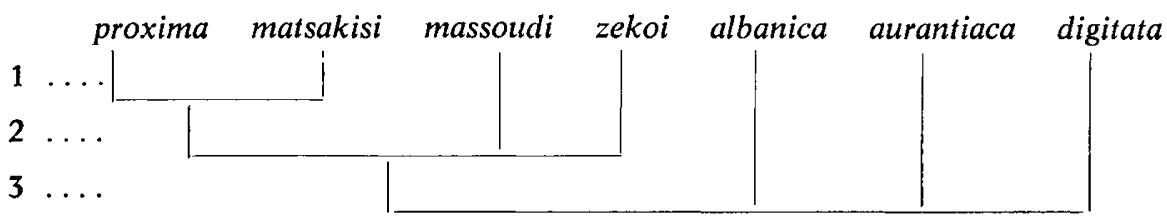

Au niveau 1 se rejoignent les espèces ayant leurs 7 chromosomes homologables. Au niveau 2, des espèces et un groupe ayant 6 chromosomes homologables. Au niveau 3 , des espèces et un groupe ayant 5 chromosomes homologables (comparaison par couples). 


\section{B. Description des caryotypes}

Une nomenclature des chromosomes - le nombre haploïde est de 7 chez toutes les espèces - a été proposée pour Bilobella massoudi en 1968, pour $B$. matsakisi en 1970 et pour $B$. aurantiaca en 1976 . Les difficultés à déterminer les homologies interspécifiques au niveau chromosomique nous ont amenés à reprendre le problème en utilisant la double nomenclature (types chromosomiques/chromosomes), dont les principes viennent d'être exposés.

\section{Bilobella aurantiaca CAROLI, 1912}

$B$. aurantiaca est l'espèce la mieux connue à ce jour. Son caryotype polytène a été étudié en détail dans différentes populations méditerranéennes par CASSAGNAU (1976), qui a pu mettre en évidence un polytypisme chromosomique remarquable. On retrouve malgré tout facilement les chromosomes homologues d'une population à l'autre, car la variabilité affecte peu la structure générale du caryotype.

\section{Bilobella massoudi CASSAgnau, 1968}

Le caryotype de cette espèce a déjà été étudié (CASSAGNAU, 1968, travail auquel on pourra se reporter pour une description détaillée). L'étendue de la variabilité chromosomique n'a point encore fait l'objet de recherches approfondies.

\section{Bilobella matsakisi CASSAGNAU, 1968}

Une précédente étude (CASSAGNaU, $1970 \mathrm{~b}$ ) illustre le caryotype de l'espèce et apporte des données importantes sur sa variabilité ; on consultera ce travail pour une description précise des chromosomes. Cette espèce se montre particulièrement polymorphe et constituera un des modèles les plus intéressants pour l'étude des variations chromosomiques.

\section{Bilobella albanica CASSAgnau \& PejA, 1979 (pl. 1)}

Nous décrirons le caryotype de cette espèce sur un matériel récolté par l'un de nous (PEJA) dans le nord de l'Albanie.

Le chromosome 1 (I) est remarquable par sa dimension (plus de 43 p. $100 \mathrm{du}$ caryotype haploïde en longueur) et la dissymétrie de ses bras qui en font un acrocentrique incontestable. On note un niveau de surcharge hétérochromatique dans la partie médiane du centromère ; les bras chromosomiques sont régulièrement structurés en bandes (270-280 bandes pour le bras long, 90-100 bandes pour le bras court).

Le chromosome 2 (II) est également acrocentrique, mais beaucoup plus court. Il possède un nodule hétérochromatique bien développé suivi d'un puff à la base du bras court, qui est ensuite structuré en bandes régulières; le bras long est entièrement structuré en bandes avec quelques ébauches de puffs peu marqués. La région centromérienne est floconneuse.

Le chromosome 5 (III) comporte un bras court faiblement structuré, muni d'un petit nodule hétérochromatique et terminé en éventail ; la région centromérienne est constituée d'une écharpe lâche, sans hétérochromatine ; le bras long, peu développé, est régulièrement structuré, avec un petit puff subterminal. 


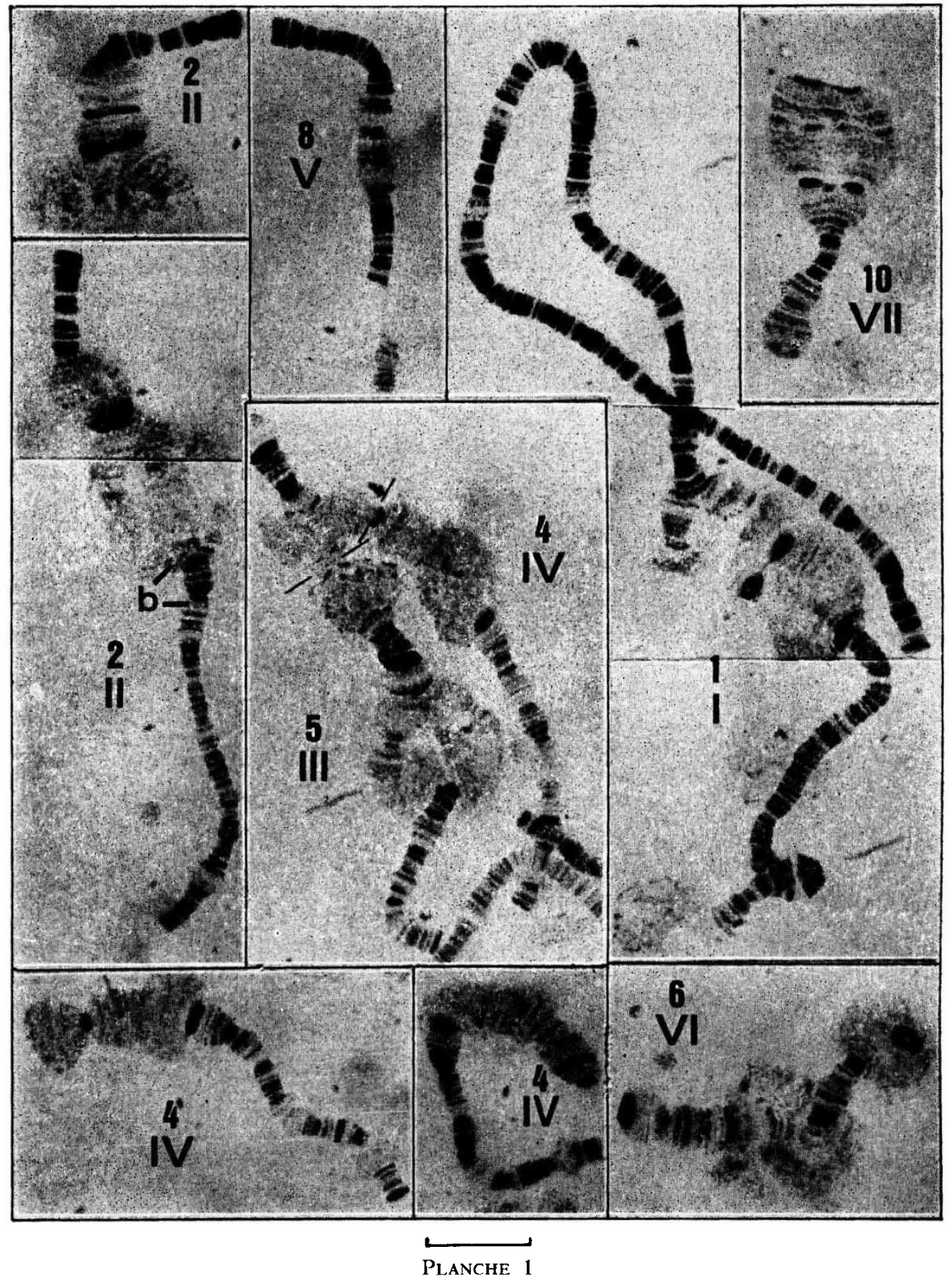

Chromosomes polytènes de Bilobella albanica (Albanie).

Polytene chromosomes of Bilobella albanica (Albany).

b : Scction du chromosome II homologue avec Bilobella proxima. Le trait représente $10 \mu \mathrm{m}$.

Section of chromosome 11 homologous with Bilobella proxima. Scale : $10 \mu \mathrm{m}$. 
Le chromosome 4 (IV) possède un bras long structuré, avec quelques ébauches de puffs, et un petit puff subterminal ; au niveau de son insertion sur l'écharpe centromérienne, on relève également un petit puff, suivi d'un nodule ovale hétérochromatique. L'écharpe centromérienne est floconneuse, et passe insensiblement à un bras court très faiblement structuré, terminé par une mince bande hétérochromatique.

Le chromosome $8(\mathrm{~V})$ est tout-à-fait inhabituel, dans la mesure où il apparaît entièrement structuré en bandes, avec un léger gonflement médian correspondant peut-être à la région centromérienne.

Le chromosome 6 (VI) est un métacentrique à 2 bras subégaux, structurés avec quelques renflements; en particulier, on observe sur chaque bras un puff terminal précédant un petit anneau hétérochromatique. La région centromérienne est dépourvue de masse hétérochromatique et entièrement constituée d'une écharpe lâchement structurée.

Le chromosome 10 (VII) comporte un court segment structuré en bandes régulières avec 2 renflements terminaux inégaux, où les bandes tendent à s'estomper. $A$ la base de l'écharpe télomérienne la plus développée est différencié un niveau hétérochromatique peu important.

Le matériel étudié est insuffisant pour déterminer l'étendue de la variabilité; on peut toutefois signaler qu'une hétérozygotie légère du bras court du chromosome II a pu être observée sur un exemplaire (pl. 1).

Les homologies avec le caryotype référence de $B$. aurantiaca ne posent pas de problèmes pour les chromosomes 1 (I), 2 (II), 5 (III) et 4 (IV), dont la structure générale est comparable entre les 2 espèces, ceci malgré le grand développement du bras long du I chez B. albanica. Par contre, le chromosome 6 (VI) de B. albanica pourrait correspondre soit à un petit V, soit à un grand VI d'aurantiaca; le B (V) d'albanica est, lui, très particulier et sans équivalent chez aurantiaca. Le 10 (VII) est très comparable au 10 (VII) des autres espèces.

Dans le détail, l'homologie des séquences de bandes entre les 2 espèces n'a pu être établie sur aucun segment chromosomique.

\section{Bilobella digitata CASSAgnaU, 1968 (pl. 2)}

L'illustration donnée sur la planche 2 a été établie d'après des exemplaires grecs du Mt Dyrfis (Ile d'Eubée).

Le chromosome 1 (I) correspond à un métacentrique relativement plus développé que chez aurantiaca (plus de 36 p. 100 de la longueur du caryotype haploïde). Les 2 bras sont régulièrement structurés, avec quelques ébauches de puffs sur le bras court. Une surcharge hétérochromatique légère peut être observée dans la partie médiane de l'écharpe centromérienne associée à une brève structuration en bandes. Un petit nodule hétérochromatique suivi d'un puff sont présents à la base du bras long.

Nous avons considéré comme chromosome 11 (II) un télocentrique du caryotype, à bras euchromatique régulièrement structuré, dépourvu de puff subterminal, et dont le centromère se poursuit par une petite houppette correspondant peut-être à un bras extrêmement court. 


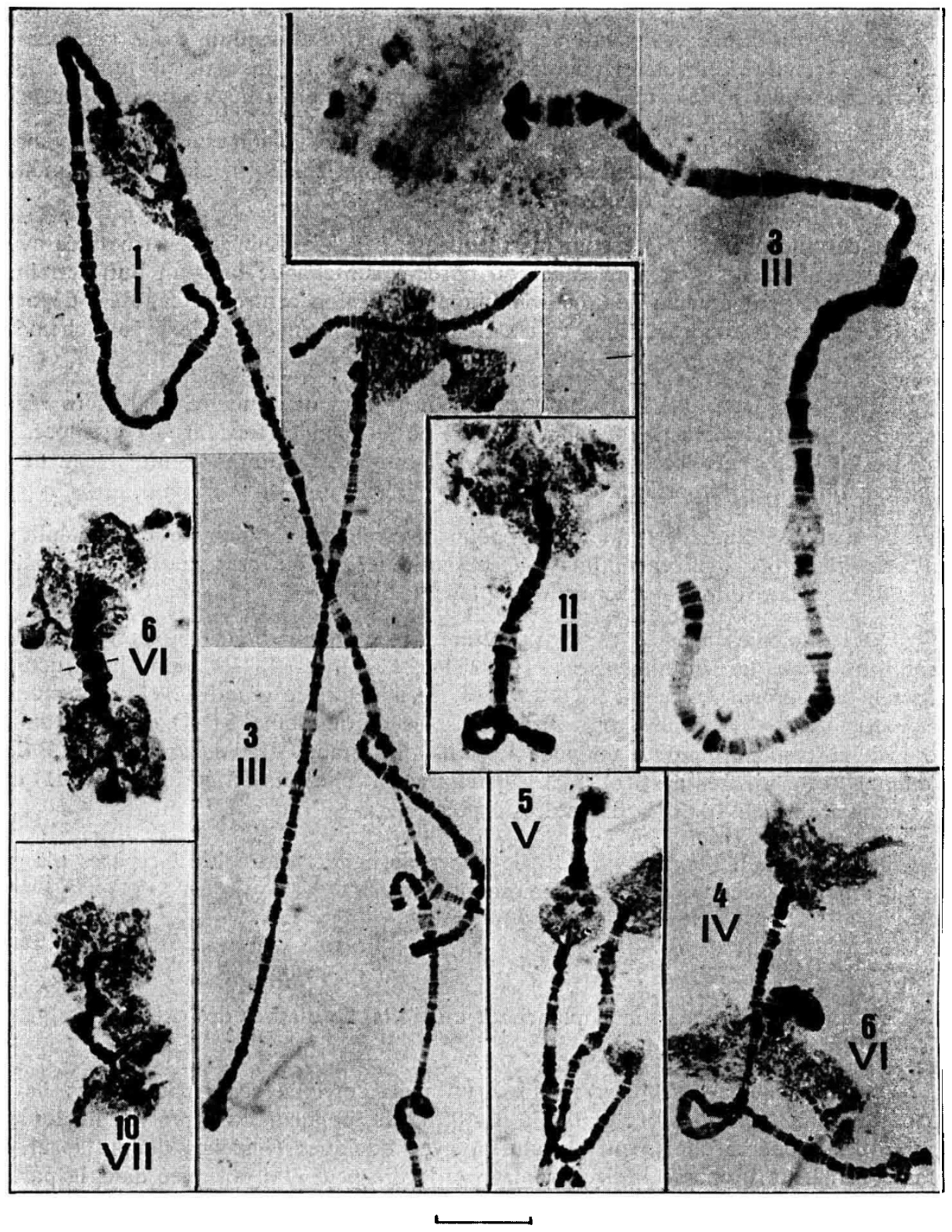

Planche 2

Chromosomes polytènes de Bilobella digitata (Grèce) et chromosome polytène III de B. zekoi, en haut à droite. Le trait représente $10 \mu \mathrm{m}$.

Polytene chromosomes of Bilobella digitata (Greece) and polytene chromosome III of B. zekoi, top right. Scale : $10 \mu \mathrm{m}$. 
Les chromosomes 3 (III) et 4 (IV) sont des télocentriques subégaux, formés chacun d'un long bras structuré avec puff subterminal. La zone centromérienne est floconneuse, munie de quelques nodules hétérochromatiques pour le 4 (IV); chez le 3 (III), elle présente une constriction subterminale suivie d'une écharpe très faiblement structurée (correspondant peut-être au bras court).

Le chromosome $5(\mathrm{~V})$ est typiquement acrocentrique; la région centromérienne présente un niveau de condensation hétérochromatique net. Les 2 bras sont entièrement structurés et terminés chacun par un puff bien développé ; on observe également quelques ébauches de puffs sur le bras long.

Les chromosomes 6 (VI) et 10 (VII) sont les plus courts du caryotype, vraisemblablement acrocentriques ou subtélocentriques. Le bras long du 6 (VI) possède plusieurs bandes hétérochromatiques, dont une terminale souvent impliquée dans des adhérences ectopiques; le centromère est floconneux, et on distingue un bras court très réduit, peu structuré, terminé par une houppette ou un petit nodule hétérochromatique (hétérozygotie). Le chromosome 10 (VII) est de longueur comparable au 6 (VI) ; son bras long comporte 2 ou 3 bandes chromophiles épaisses et quelques autres plus ou moins effacées ; il se termine en éventail faiblement structuré. Le centromère est floconneux, et possède à son extrémité un petit nodule hétérochromatique surmonté d'une houppette très courte (correspondant peut-être au bras court).

La comparäison avec $B$. aurantiaca montre d'importantes divergences dans la structure générale des 2 caryotypes. En fait, $B$. digitata s'écarte de toutes les autres espèces par la présence d'un long télocentrique supplémentaire 11 (II), dont l'origine ne peut provenir que d'un remaniement chromosomique important que nous n'avons pas su préciser. Il est vrai que l'espèce est morphologiquement bien particulière par l'élongation remarquable de ses tubercules postérieurs, et pourrait appartenir à un groupe isolé du genre Bilobella.

\section{Bilobella proxima Cassagnau \& Peja, 1979 (pl. 3)}

La description correspond à un exemplaire du Vermosh (nord de l'Albanie) récolté par PEJA.

Le chromosome 1 (I) est un métacentrique bien développé, dont les 2 bras, peu inégaux, sont structurés sur toute leur longueur; on observe un gros puff près de la base du bras court et plusieurs puffs plus réduits sur les 2 bras; on notera en particulier un renflement terminal sur le bras court, et un puff subterminal sur le bras long. L'écharpe centromérienne est munie d'une mince bande hétérochromatique médiane.

Le chromosome 2 (II) est typiquement acrocentrique. Le bras long, bien structuré, se termine par une large houppette floconneuse et porte 2 ou 3 renflements sur sa longueur; le bras court présente 2 petits puffs successifs dans sa partie proximale, et un léger renflement subterminal ; l'écharpe centromérienne est dépourvue de masses hétérochromatiques.

Le chromosome 3 (IV) est un télocentrique de grande taille. Son bras est régulièrement structuré, à l'exception d'un double puff proximal et d'un puff subterminal bien développé ; l'écharpe centromérienne renferme quelques granules chromophiles, et se termine par un nodule hétérochromatique peu dense surmonté d'une petite houppette. 


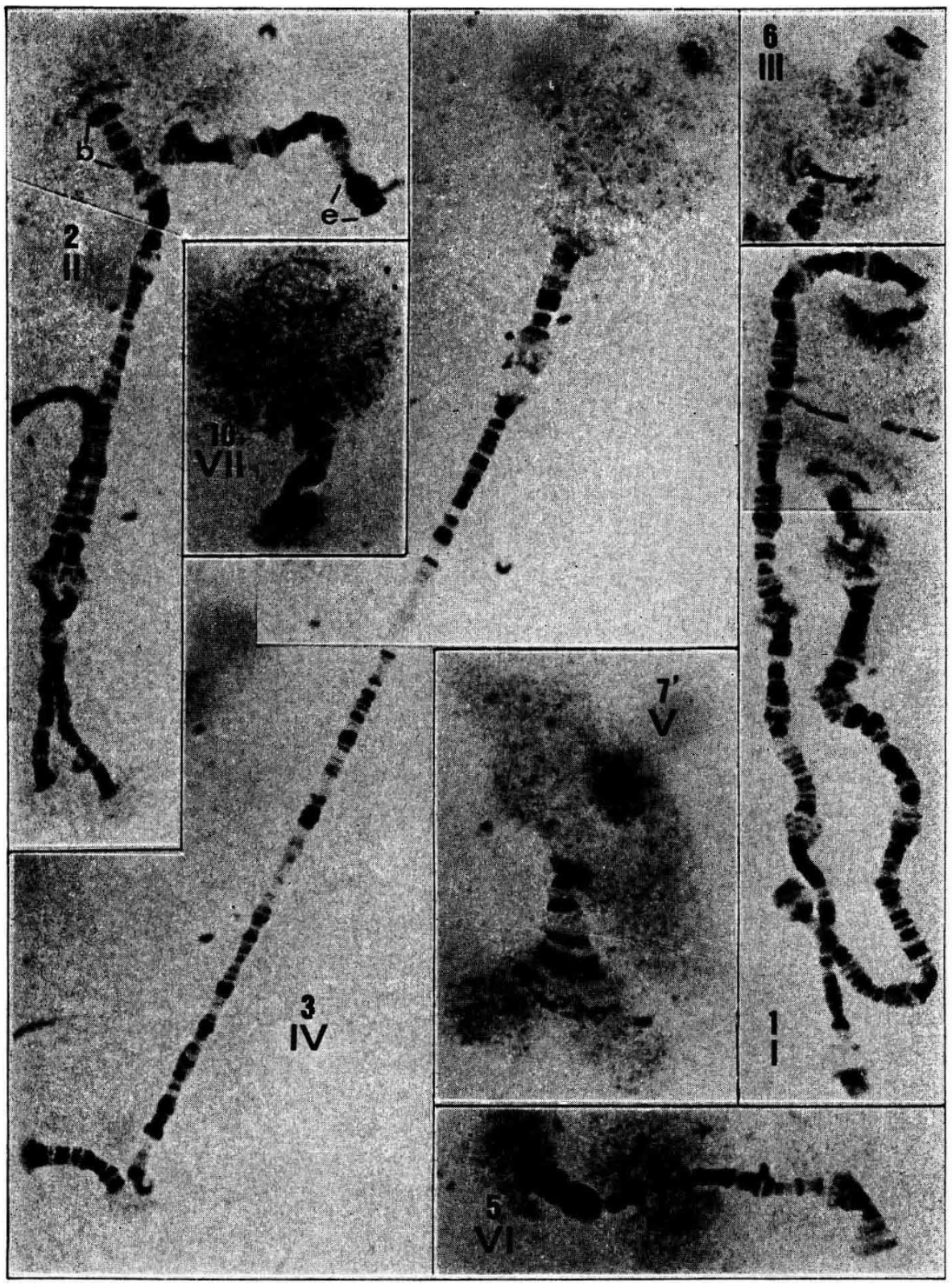

Planche 3

Chromosomes polytènes de Bilobella proxima (Albanie).

Polytene chromosomes of Bilobella proxima (Albany).

b : Section du chromosome II homologue avec B. albanica.

Section of chromosome II homologous with Bilobella albanica.

e : Section du chromosome II homologue avec B. matsakisi. Le trait représente $10 \mu \mathrm{m}$. Section of chromosome II homologous with B. matsakisi. Scale : $10 \mathrm{\mu m}$. 
Le chromosome 5 (VI) est assez semblable à celui de $B$. aurantiaca; il possède 2 bras structurés, l'un court et très chromophile ( imprégnation hétérochromatique »), l'autre long, pourvu d'un petit puff à son tiers distal et terminé par une houppette faiblement structurée. L'écharpe centromérienne est floconneuse, avec quelques petites masses hétérochromatiques.

Le chromosome 6 (III) est constitué d'une large zone centrale floconneuse, d'un bras structuré très court et conique, et d'un bras également très court, large et floconneux, muni de 2 bandes terminales et rattaché à l'écharpe centromérienne au niveau d'une constriction marquée par un petit nodule hétérochromatique.

Le chromosome 10 (VII) est un télocentrique beaucoup plus court que le 3 (IV); la région centromérienne terminale est floconneuse, avec quelques bandes hétérochromatiques. Le bras euchromatique structuré possède un renflement proximal et une large écharpe floconneuse terminale.

Le chromosome $7^{\prime}(\mathrm{V})$ est très diffus; on y observe une large écharpe floconneuse à chacune des 2 extrémités, le segment intermédiaire étant faiblement structuré avec 6 à 8 bandes chromophiles.

Par rapport au caryotype de B. aurantiaca, celui de $B$. proxima se caractérise au niveau de la structure générale par la présence d'un unique long télocentrique : 3 (IV). Aucune homologie précise dans les séquences de bandes n'a pu être mise en évidence entre les 2 espèces.

Le caryotype de $B$. proxima présente par contre de fortes similitudes dans sa structure générale avec celui de $B$. matsakisi, espèce morphologiquement proche (Cassagnau, $1970 \mathrm{~b}$ et pl. 5).

\section{Bilobella zekoi Cassagnau \& Peja (P1. 2 et 4)}

Cette espèce a été récoltée par PEJA dans le nord de l'Albanie; c'est sur ce matériel qu'a pu être établi le caryotype.

Comme chez B. proxima ou matsakisi, on a chez B. zekoi 3 chromosomes longs (chacun représentant 15 p. 100 minimum de la longueur du caryotype haploïde) s'opposant à 4 chromosomes courts (dont aucun ne dépasse 7 p. 100 de la longueur du caryotype haploïde) (tabl. 2).

Le chromosome 1 (I) est un métacentrique constitué de 2 bras assez inégaux, régulièrement structurés, portant quelques ébauches de puffs, dont un renflement terminal pour le bras court ; l'écharpe centromérienne est dépourvue de masses hétérochromatiques.

Le chromosome 2 (II) (voir pl. 4) est également un métacentrique à bras inégaux, régulièrement structurés ; l'écharpe centromérienne est semblable à celle du I ; bras court et bras long portent chacun 4-5 renflements peu marqués; le bras long possède en outre un puff terminal associé à un petit nodule hétérochromatique.

Le chromosome 3 (III) (voir pl. 2) est un télocentrique ou subtélocentrique de grande taille. Le bras long, bien structuré, présente plusieurs ébauches de puffs, dont aucune n'est subterminale, et une forte bande hétérochromatique à sa base. L'écharpe centromérienne floconneuse est surmontée par une houppette, elle-même terminée par une petite masse hétérochromatique (bras court ?). 


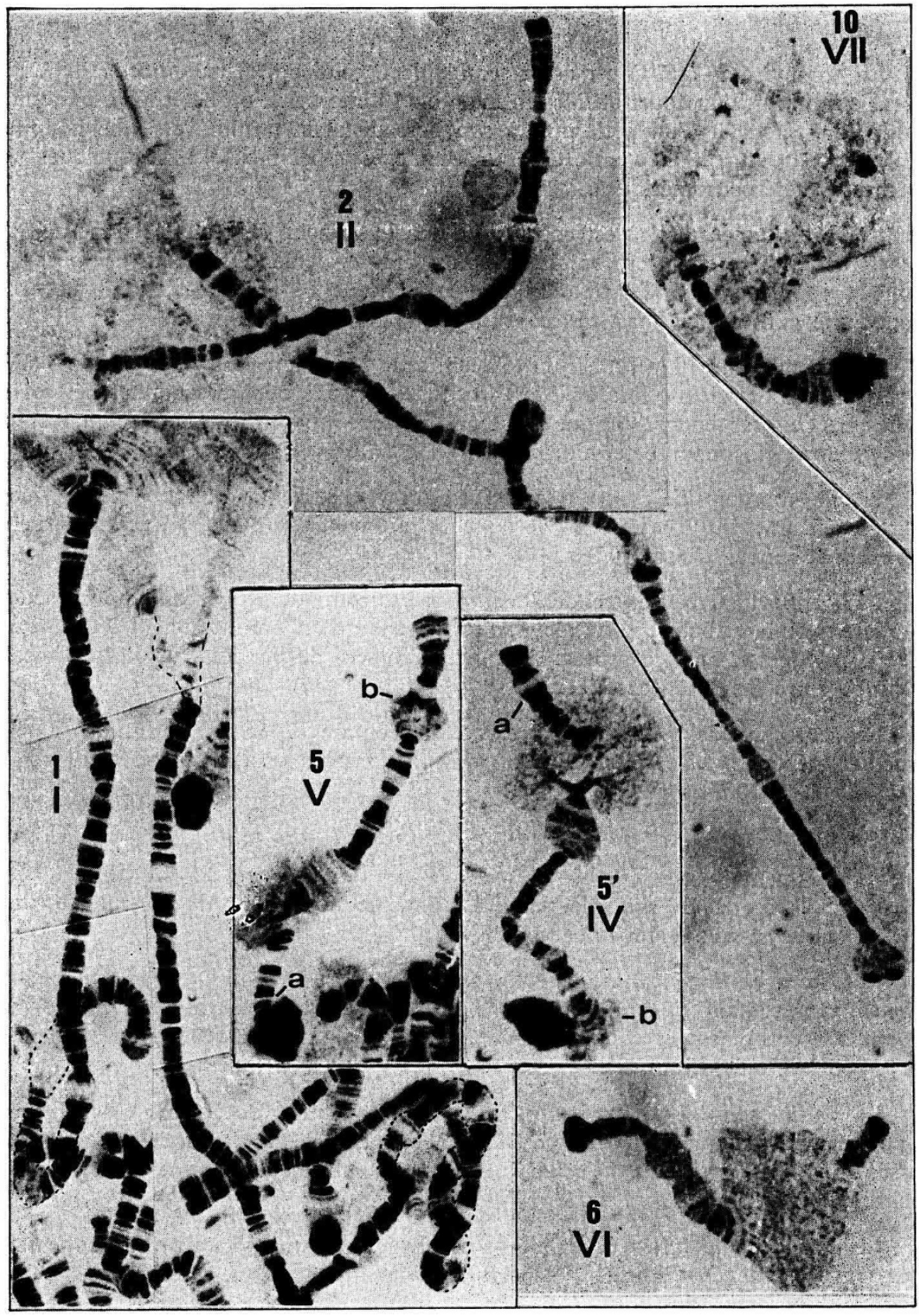

Planche 4

Chromosomes polytènes de Bilobella zekoi (Albanie); le chromosome III est représenté sur la planche 2. Le trait représente $10 \mu \mathrm{m}$. Polytene chromosomes of Bilobella zekoi (Albany); chromosome III is figured on plate 2. Scale : $10 \mu \mathrm{m}$. 
On remarque une étonnante similitude entre les chromosomes 5 (V) et $5^{\prime}$ (IV), tous deux acrocentriques. Ils sont en effet constitués d'un bras court, d'une zone centromérienne floconneuse et d'un bras long muni à sa base d'un gros puff ovoïde et d'un second dans son tiers ou son quart distal. La séquence des bandes de la partie médiane du bras long semble presque identique sur les 2 chromosomes. Une différence essentielle tient à ce que la partie distale du bras long, structurée normalement pour le $\mathrm{V}$, prend l'aspect d'une large masse irrégulière d'hétérochromatine, avec quelques bandes vestigiales, pour le chromosome IV. L'interprétation de cette similitude a été précisée plus haut (inversion péricentrique du segment $a-b$ ).

Le chromosome 6 (VI) comporte une écharpe centromérienne sans surcharge hétérochromatique et 2 bras très inégaux (acrocentriques). Le bras long débute par 2 gros renflements (puffs ?) successifs, suivis par une autre section structurée ; il se termine par un nodule hétérochromatique. Le bras court comporte moins de 10 bandes, avec également un petit nodule hétérochromatique à son extrémité.

Le chromosome 10 (VII) est un télocentrique formé d'une écharpe centromérienne floconneuse de grande taille, terminée par un nodule hétérochromatique; le bras long est bien structuré sur sa moitié proximale, mais présente 2 gros renflements successifs sur sa moitié distale; il se termine par une masse ovoïde d'hétérochromatine.

Le caryotype de $B$. zekoi - espèce morphologiquement et chétotaxiquement très évoluée - se caractérise par la similitude des chromosomes 5 (V) et $5^{\prime}$ (IV), phénomène qui n'a été retrouvé chez aucune des autres espèces examinées. Là encore, les recherches d'homologies interspécifiques au niveau des séquences de bandes ont été vaines.

\section{Discussion}

\section{A. Approche du problème par l'étude morphométrique des chromosomes}

Etant donné la variabilité extrême observée dans les séquences de bandes, nous avons abordé le problème des affinités spécifiques par la prise en compte de 2 critères :

- Structure générale du caryotype définie par la longueur relative de chaque chromosome (par rapport au caryotype haploïde complet) et par le type, soit télo-, soit acro- ou métacentrique.

- Histogramme de longueur des 6 bras chromosomiques les plus développés.

\section{Structure générale du caryotype}

La longueur relative des différents chromosomes varie beaucoup d'une forme à l'autre (tabl. 2). Il se dégage cependant 3 groupes d'espèces :

a) zekoi, matsakisi et proxima possèdent 4 chromosomes courts (chacun mesurant moins de 10 p. 100 du caryotype haploïde) s'opposant à 3 chromosomes longs ( 2 méta- ou acrocentriques, 1 télo- ou subtélocentrique, chacun dépassant 15 p. 100 du caryotype haploïde). 
TABleau 2

Morphométrie des chromosomes chez les Bilobella étudiés.

Morphometry of the chromosomes in the Bilobella species under study.

\begin{tabular}{|c|c|c|c|c|}
\hline & \multicolumn{3}{|c|}{$\begin{array}{c}\text { Base } 100=\text { longueur du bras long } \\
\text { du chromosome I }\end{array}$} & \multirow{2}{*}{$\begin{array}{c}\text { Longueur } \\
\text { du chromosome } \\
\text { par rapport } \\
\text { à la longueur } \\
\text { du caryotype } \\
\text { haplö̈de } \\
\text { (en pourcentage) }\end{array}$} \\
\hline & \multicolumn{2}{|c|}{$\begin{array}{l}\text { Bras long / bras court } \\
\text { (région centromérienne) }\end{array}$} & $\begin{array}{l}\text { Chromosome } \\
\text { complet }\end{array}$ & \\
\hline \multirow[t]{2}{*}{ aurantiaca (Gredos) } & $\begin{array}{ll}1 & \text { (I) } \\
2 & (\text { II) } \\
3 & (\text { III) } \\
4 & (\text { IV) } \\
5 & \text { (V) } \\
6 & \text { (VI) } \\
7 & \text { (VII) }\end{array}$ & $\begin{aligned} & 100 / 76(14) \\
& 92 / 58(14) \\
& 60(20) \\
& 58(18) \\
& 42 / 16(14) \\
& 40 \\
& 32\end{aligned}$ & $\begin{array}{r}190 \\
164 \\
80 \\
74 \\
72 \\
40 \\
32\end{array}$ & $\begin{array}{r}29,1 \\
25,1 \\
12,3 \\
11,3 \\
11,0 \\
6,2 \\
5,0\end{array}$ \\
\hline & & & 652 & \\
\hline \multirow[t]{2}{*}{ albanica } & $\begin{aligned} 1 & (\text { I) } \\
2 & (\text { II) } \\
5 & (\text { III) } \\
4 & (\text { IV) } \\
8 & (\mathrm{~V}) \\
6 & (\mathrm{VI}) \\
10 & (\mathrm{VII})\end{aligned}$ & $\begin{array}{rr}100 / 35 & (15) \\
30 / 10 & (17) \\
14 / 12 & (9) \\
21 / 4 & (7) \\
25 & \\
10 / 10 & (7) \\
20 & \end{array}$ & $\begin{array}{r}150 \\
57 \\
35 \\
32 \\
27 \\
25 \\
20\end{array}$ & $\begin{array}{r}43,4 \\
16,5 \\
10,1 \\
9,2 \\
7,8 \\
7,2 \\
5,8\end{array}$ \\
\hline & & & 346 & \\
\hline \multirow[t]{2}{*}{ massoudi } & $\begin{aligned} 1 & (\mathrm{I}) \\
2 & (\mathrm{II}) \\
5 & (\mathrm{III}) \\
9 & (\mathrm{~V}) \\
3 & (\mathrm{IV}) \\
6 & (\mathrm{VI}) \\
10 & (\mathrm{VII})\end{aligned}$ & $\begin{aligned} 100 / 81 & (12) \\
85 / 35 & (15) \\
42 / 30 & (23) \\
66 & (18) \\
55 & (22) \\
27 / 23 & (12) \\
30 & (23)\end{aligned}$ & $\begin{array}{r}193 \\
135 \\
95 \\
84 \\
77 \\
62 \\
53\end{array}$ & $\begin{array}{r}27,6 \\
19,3 \\
13,6 \\
12,0 \\
11,0 \\
8,9 \\
7,6\end{array}$ \\
\hline & & & 699 & \\
\hline \multirow[t]{2}{*}{ digitata $\ldots \ldots \ldots \ldots$} & $\begin{aligned} 1 & (\mathrm{I}) \\
3 & (\mathrm{III}) \\
4 & (\mathrm{IV}) \\
5 & (\mathrm{~V}) \\
11 & (\mathrm{II}) \\
6 & (\mathrm{VI}) \\
10 & (\mathrm{VII})\end{aligned}$ & $\begin{array}{rr}100 / 76 & (12) \\
96 & (17) \\
70 & (10) \\
39 / 12 & (7) \\
41 & (10) \\
9 / 7 & (5) \\
7 & (7)\end{array}$ & $\begin{array}{r}188 \\
109 \\
80 \\
58 \\
51 \\
21 \\
14\end{array}$ & $\begin{array}{r}36,1 \\
20,9 \\
15,4 \\
11,1 \\
9,8 \\
4,0 \\
2,7\end{array}$ \\
\hline & & & 521 & \\
\hline
\end{tabular}


TABleau 2 (suite)

\begin{tabular}{|c|c|c|c|c|}
\hline & \multicolumn{3}{|c|}{$\begin{array}{c}\text { Base } 100=\text { longueur du bras long } \\
\text { du chromosome I }\end{array}$} & \multirow{2}{*}{$\begin{array}{c}\text { Longueur } \\
\text { du chromosome } \\
\text { par rapport } \\
\text { à la longueur } \\
\text { du caryotype } \\
\text { haploïde } \\
\text { (en pourcentage) }\end{array}$} \\
\hline & \multicolumn{2}{|c|}{$\begin{array}{l}\text { Bras long / bras court } \\
\text { (région centrom ́́rienne) }\end{array}$} & $\begin{array}{c}\text { Chromsome } \\
\text { complet }\end{array}$ & \\
\hline \multirow[t]{2}{*}{ proxima } & $\begin{aligned} 1 & (\mathrm{I}) \\
3 & (\mathrm{IV}) \\
2 & (\mathrm{II}) \\
5 & (\mathrm{VI}) \\
7^{\prime} & (\mathrm{V}) \\
10 & (\mathrm{VII}) \\
6 & (\mathrm{III})\end{aligned}$ & $\begin{array}{rr}100 / 74 & (16) \\
89 & (21) \\
68 / 26 & (13) \\
24 / 13 & (8) \\
32 & \\
16 & (10) \\
8 / 6 & (8)\end{array}$ & $\begin{array}{r}190 \\
110 \\
107 \\
45 \\
32 \\
26 \\
22\end{array}$ & $\begin{array}{r}35,7 \\
20,7 \\
20,1 \\
8,5 \\
6,0 \\
4,9 \\
4,1\end{array}$ \\
\hline & & & 532 & \\
\hline \multirow[t]{2}{*}{ matsakisi } & $\begin{aligned} 1 & (\mathrm{I}) \\
2 & (\mathrm{II}) \\
3 & (\mathrm{IV}) \\
7^{\prime} & (\mathrm{V}) \\
5 & (\mathrm{VI}) \\
10 & (\mathrm{VII}) \\
6 & (\mathrm{III})\end{aligned}$ & $\begin{array}{rr}100 / 87 & (22) \\
61 / 28 & (17) \\
65 & (20) \\
40 & \\
16 / 8 & (8) \\
13 & (11) \\
24 & \end{array}$ & $\begin{array}{r}209 \\
106 \\
85 \\
40 \\
32 \\
24 \\
24\end{array}$ & $\begin{array}{r}40,2 \\
20,4 \\
16,3 \\
7,7 \\
6,2 \\
4,6 \\
4,6\end{array}$ \\
\hline & & & 520 & \\
\hline \multirow[t]{2}{*}{ zekoi } & $\begin{aligned} 1 & (\mathrm{I}) \\
2 & (\mathrm{II}) \\
3 & (\mathrm{III}) \\
5^{\prime} & (\mathrm{IV}) \\
5 & (\mathrm{~V}) \\
10 & (\mathrm{VII}) \\
6 & (\mathrm{VI})\end{aligned}$ & $\begin{array}{rr}100 / 61 & (13) \\
65 / 39 & (10) \\
68 & (10) \\
21 / 8 & (3) \\
19 / 8 & (3) \\
16 & (10) \\
11 / 3 & (6)\end{array}$ & $\begin{array}{r}174 \\
114 \\
78 \\
32 \\
30 \\
26 \\
20\end{array}$ & $\begin{array}{r}36,7 \\
24,1 \\
16,5 \\
6,8 \\
6,3 \\
5,4 \\
4,2\end{array}$ \\
\hline & & & 474 & \\
\hline
\end{tabular}

b) Chez aurantiaca et massoudi, le chromosome le plus long reste relativement. peu développé puisqu'il n'atteint pas 30 p. 100 de la longueur du caryotype haploïde (35 p. 100 au minimum pour celui des autres espèces). Chez chacune de ces 2 espèces, 5 chromosomes dépassent 10 p. 100 du caryotype haploïde.

c) albanica est caractérisé par la forte disproportion entre le chromosome I très. long, et les autres chromosomes dont aucun ne dépasse 20 p. 100 du caryotype haploïde.

d) digitata montre un caryotype intermédiaire entre ceux des groupes a et b en ce qui concerne les longueurs relatives des chromosomes. 
On peut encore souligner que $B$. massoudi, digitata et aurantiaca ont toutes une paire de télo- ou subtélocentriques de longueur très comparable. Il s'agit des chromosomes 3 (IV) et 9 (V) pour B. massoudi, et des chromosomes 3 (III) et 4 (IV) pour les autres espèces.

La partition des espèces opérée sur le critère des longueurs relatives des chromosomes s'accorde donc assez bien avec les données tirées de la chétotaxie du phénotype externe exposé sur la figure 2. Elle rapproche toutefois aurantiaca et massoudi, qui sont par ailleurs les deux espèces les plus occidentales du genre parmi celles étudiées ici.

\section{Longueur relative des bras chromosomiques (fig. 4)}

En donnant la valeur 100 au bras long du chromosome I, il est possible de donner une mesure relative des autres bras chromosomiques. Nous nous sommes limités pour le caryotype de chaque espèce aux 6 bras chromosomiques les plus développés, ce qui donne le diagramme représentatif de la figure 4. L'éventail et le type de distribution des valeurs obtenues sont très variables selon l'espèce considérée.

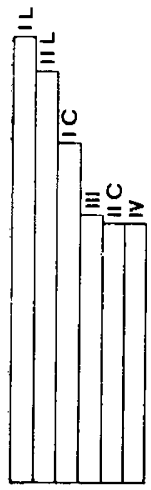

aurantiace

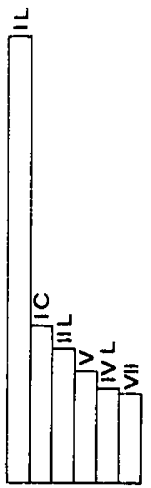

albanica

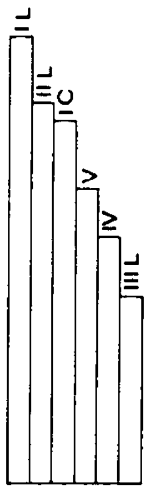

massoudi

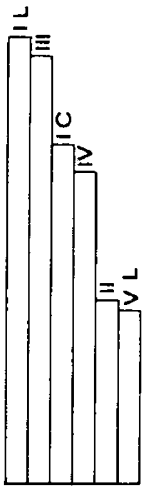

digitata

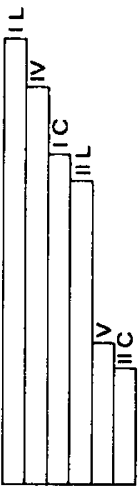

proxima

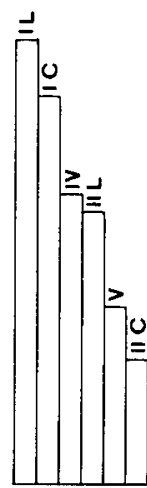

matsakisi

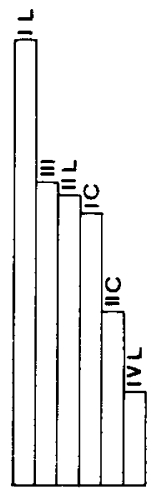

zekoi

FIG. 4

Histogrammes des longueurs relatives (rapportées au bras le plus long : IL) des 6 bras chromosomiques les plus développés chez les 7 espèces de Bilobella étudiées.

Chaque bras chromosomique est identifié par le numéro du chromosome suivi le cas échéant de L (bras long) ou C (bras court).

Histogram of the relative lengths of the 6 largest chromosomal arms related to the longest one (IL) in the 7 species of Bilobella under study. Each chromosomal arm is named by the chromosome number eventually associated with $L$ (long arm) or $C$ (short arm).

Les espèces, classées par ordre croissant d'écart entre les longueurs des bras chromosomiques le plus court et le plus long, se rangent ainsi : aurantiaca-massoudidigitata-matsakisi-proxima-zekoi-albanica. Si l'on excepte $B$. albanica, on retrouve donc une distribution proche de celle exposée dans le paragraphe précédent, avec un 


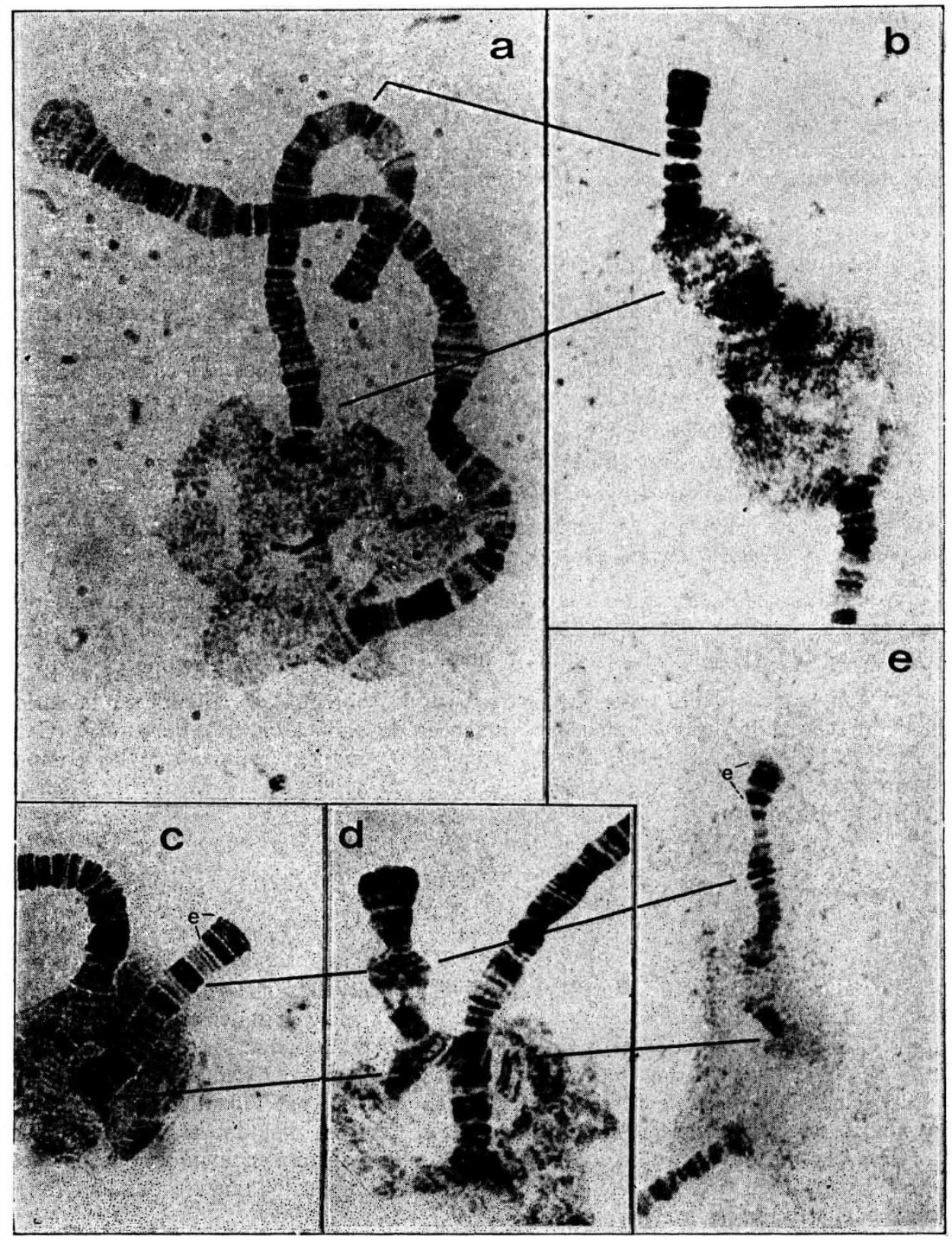

Planche 5

Bras court du chromosome II chez différentes espèces de Bilobella. Short arm of chromosome II in different species of Bilobella.
a : B. aurantiaca (Gredos).
B. aurantiaca (Gredos).
b : B. albanica (Albanie).
B. albanica (Albany).
c : B. matsakisi (Parnasse).
B. matsakisi (Parnasse).

d : B. massoudi (Slovénie).

B. massoudi (Slovénie).

e : B. proxima (Albanie).

B. proxima (Albany).

Nous avons indiqué des homologies possibles de «zones» chromosomiques en traits continus. Le trait représente $10 \mu \mathrm{m}$. $10 \mu \mathrm{m}$.

We indicated possible homologies of chromosomal bands in continuous line. Scale : 
écart croissant des longueurs de bras chromosomiques allant des espèces à chétotaxie peu abondante, vers les espèces plurichaetotiques.

\section{B. Variations interspécifiques dans les séquences de bandes}

Nous avons dit plus haut qu'il était très difficile d'établir les homologies interspécifiques entre séquence de bandes. Ceci tient vraisemblablement à ce que ces séquences sont complètement différentes dans la majorité des cas. Un examen détaillé des caryotypes nous a permis de retrouver des séquences comparables entre 2 espèces dans un petit nombre de cas :

- Bras court du chromosome 2 (II) entre B. proxima et B. matsakisi : l'extrémité «e " identique dans les 2 espèces (pl. 5).

- Base du bras long du chromosome 2 (II) : elle semble identique entre $B$. proxima et $B$. albanica (b, pl. 1 et 3), peut-être aussi chez matsakisi.

Nous n'avons aucun autre cas d'homologie interspécifique nette au niveau des séquences de bandes. Cette situation a été illustrée sur la planche 5 , au niveau des bras courts du 2 (II) chez B. aurantiaca, albanica, matsakisi, massoudi et proxima.

Si l'on rapproche ces observations des résultats obtenus par ailleurs sur la variabilité intraspécifique très étendue des séquences de bandes chez $B$. aurantiaca (CASSagnau, 1976 ; Dallai, 1979 ; Deharveng, 1982), on peut estimer que les comparaisons interspécifiques à ce niveau ne peuvent en aucun cas permettre de préciser la phylogenèse du genre.

\section{Conclusions}

L'analyse comparée des caryotypes polytènes salivaires chez 7 espèces de Bilobella de la région méditerranéenne nous révèle des structures tellement dissemblables qu'il est difficile pour la plupart des chromosomes de prononcer des analogies irréfutables d'une espèce à l'autre. Les analogies quand elles existent portent plus sur l'allure générale et la taille des bras chromosomiques que sur l'identité des séquences de bandes chromophiles et des puffs éventuels. Ceci est d'autant plus remarquable que les espèces de ce genre ne se distinguent, sur le plan morphologique, que par des caractères tégumentaires ou chétotaxiques parfois discrets qui ont été pendant longtemps négligés par les systématiciens.

Nous nous trouvons donc en présence de différences peu marquées au niveau du phénotype externe, mais sous-tendues par des différences considérables au niveau chromosomique, différences dont l'origine n'a pas pu être précisée, car elles n'ont pu être nettement rapportées pour l'instant a aucun remaniement de type classique (inversion, translocation ou délétion). Par-là même, l'analyse des caryotypes, aussi fine soit-elle, n'a pas permis jusqu'à présent de préciser les affinités phylogénétiques au sein du genre. Ajoutons qu'un tel état de fait n'est pas particulier aux Bilobella mais se retrouve dans la plupart des genres de Neanurinae analysés à ce jour, ce qui rend illusoire les tentatives de cytogénétique taxinomique dans ce groupe. Une telle situation diffère donc profondément de celle rencontrée chez les Diptères Simuliides, Drosophiles ou Chironomides particulièrement bien étudiés sous cet angle. Dans ces grou- 
pes, des espèces voisines se distinguent au niveau du caryotype par un nombre restreint de remaniements classiques, certaines étant même homoséquentielles.

Une telle stabilité permet évidemment des reconstitutions phylogénétiques cohérentes, devenues classiques chez les Diptères.

Nous formulerons deux hypothèses susceptibles de rendre compte de la diversité de structure des caryotypes au sein du genre Bilobella et plus généralement des Neanurinae.

Si on estime que les espèces définies au niveau morphologique correspondent à de bonnes espèces, on est alors amené à se demander dans quelle mesure le caryotype est bien le reflet de leur génotype. La variabilité chromosomique très importante mise en évidence par ailleurs entre populations d'une même espèce (CASSAGNAU, 1976) ou au sein d'une même population (DEHARveng, 1982) chez Bilobella aurantiaca vient également à l'appui d'une conception dissociant jusqu'à un certain point le "phénotype chromosomique " (cf. LIMA DE FARIA, 1983) du phénotype externe et du génotype.

Une seconde hypothèse consisterait à tenir le caryotype observé comme une image relativement fidèle du génotype. Les profondes différences chromosomiques relevées entre les espèces du genre Bilobella correspondraient alors à des différences génétiques réelles qui s'exprimeraient au contraire de façon atténuée au niveau du phénotype morphologique externe. Dans cette éventualité le polytypisme chromosomique observé chez Bilobella aurantiaca (CASSAGNAU, 1976) résulterait de l'existence d'un grand nombre d' "espèces biologiques cryptiques" au sein de la même "espèce " morphologique. L'intensité du polymorphisme chromosomique mis en évidence dans certaines populations (DEHARVENG, 1982) ne permet guère toutefois de pousser cette seconde hypothèse jusque dans ses implications ultimes, d'autant plus que nous n'avons pour l'instant aucune donnée sur les critères d'hybridation, celle-ci étant fort difficile à réaliser dans ce groupe d'insectes.

En tout état de cause il serait hasardeux d'attacher à cette extraordinaire diversité du phénotype chromosomique la même signification évolutive que dans d'autres groupes taxinomiques à structures stabilisées.

On est en droit de se demander alors si ces divergences de la garniture polytène sont bien le reflet fidèle de la distance génétique des espèces au sein d'un même genre. Peut-être peut-on y voir aussi la conséquence de la plasticité du génome, de l'accumulation au cours de l'évolution d'effets dus à la présence d'éléments mobiles dispersés produisant des arrangements chromosomiques liés au mécanisme de transposition (inversions, délétions, translocations) (BREGLIANO, 1983), trop nombreux pour être aisément décelables.

Quoiqu'il en soit, s'il est possible d'envisager chez les Collemboles Neanurinae, l'utilisation de la variabilité chromosomique au sein des espèces et des populations en vue de mieux comprendre les processus de l'adaptation aux conditions du milieu (en particulier par l'analyse des fluctuations de l'hétérochromatine) il ne semble pas que l'on puisse espérer tirer d'utiles renseignements phylogénétiques de l'étude des caryotypes polytènes, diversifiés au point qu'on ne peut plus reconnaître les chromosomes, ou les bras chromosomiques, homologues au sein d'un même genre. 


\section{Références bibliographiques}

Bregliano J.C.. 1983. Les gènes sauteurs. Le courrier du C.N.R.S., 51, 19-24.

Cassagnau P., 1968 a. Sur la structure des chromosomes salivaires de Bilobella massoudi Cassagnau (Collembole Neanuridae). Chromosoma, 24, 42-58.

Cassagnau P., 1968 b. Les espèces européennes du genre Bilobella (Collembole Neanuridae). Bull. Mus. Natl. Hist. Nat., 40, 292-307.

CASSAGNAU P., 1970 a. Inversions péricentriques et polygénotypisme dans une population de Bilobella (Collembole) du Mont Parnasse (Grèce). C.R. Acad. Sci., 270, 529-532.

Cassagnau P., 1970 b. Sur les chromosomes salivaires de Bilobella matsakisi Cass. (Collembole Neanuridae) du col de Katara (Grèce). Biol. Gallo-hellen., 3, 71-80.

Cassagnau P., 1975. Le polymorphisme des chromosomes polytènes de Bilobella aurantiaca Caroli (Collembole) et sa signification biogéographique et écologique. C.R. Acad. Sci., 280, 2777-2780.

Cassagnau P., 1976. La variabilité des chromosomes polytènes chez Bilobella aurantiaca (Collembole Neanuridae) et ses rapports avec la biogéographie et l'écologie de l'espèce. Arch. Zool. Exp. Gén., 117. 511-572.

Cassagnau P., 1979. Les Collemboles Neanuridae des pays Dinaro-balkaniques : leur intérêt phylogénétique et biogéographique. $1^{\text {er }}$ Symposium international sur la zoogéographie et l'écologie de la Grèce et des régions avoisinantes. Athènes, avril 1978. Biol. Gallo-hellen., 8, 185-204.

Cassagnau P., Peja N., 1979. Diagnoses préliminaires de quelques Neanuridae de Grèce et d'Albanie. $1^{\mathrm{rr}}$ Symposium international sur la zoogéographie et l'écologie de la Grèce et des régions avoisinantes. Athènes, avril 1978. Biol. Gallo-hellen., 8, 205-222.

DallaI R., 1979. Polytene chromosomes of some Bilobella aurantiaca (Collembolla) Italian populations. Bull. Zool., 46, 231-249.

Deharveng L., 1982. Polymorphism of polytene chromosomes in Bilobella aurantiaca (Insecta : Collembola). Study of a population from Sierra de Gredos (Central Spain). Chromosoma (Berl.), 85, 201-214.

Deharveng L., 1982. Contribution à l'étude des Collemboles Neanurinae : évolution, spéciation, polymorphisme somatique et chromosomique des formes européennes, $284 \mathrm{pp}$. Thèse d'Etat, Toulouse.

Deharveng L., Lee B.H., 1984. Polytene chromosomal variability of Bilobella aurantiaca (Collembola) from Sainte-Baume population (France). Caryologia, 37, 51-67.

Dunbar R.W., 1959. The salivary gland chromosomes of seven forms of black flies included in Eusimulium aureum Fries. Can. J. Zool., 37, 495-525.

KeYL H.G., 1960. Die cytologische Diagnostik der Chironomiden. II. - Diagnosen der Geschwisterarten Chironomus acidophilus n.sp. und Ch. uliginosus n.sp. Arch. Hydrobiol., 57, 187-195.

KeYL H.G., 1961. Die cytologische Diagnostik der Chironomiden. III. - Diagnose von Chironomus parathummi n.sp. und Ergänzungen zur Bestimmungstabelle. Arch. Hydrobiol., 58, 1-6.

KeYL H.G., KeYl I., 1959. Die cytologische Diagnostik der Chironomiden. I. - Bestimmungstabelle für die Gattung Chironomus auf Grund der Speicheldrüsen-Chromosomen. Arch. Hydrobiol., 56, 43-57.

Lemeunier F., 1979. Phylogénie des espèces de Drosophila du sous-groupe melanogaster analysée par des méthodes caryologiques et génétiques, $213 \mathrm{p}$. Thèse d'Etat, Paris VI.

LIMA DE FARIA A., 1983. Molecular evolution and organization of the chromosome, 1186 pp. Elsevier édit., Amsterdam. 